\title{
Managing local dependencies in asymptotic theory for maxima of stationary random fields
}

\author{
Adam Jakubowski ${ }^{1} \cdot$ Natalia Soja-Kukieła $^{1}$ (D)
}

Received: 15 January 2018 / Revised: 17 September 2018 / Accepted: 18 September 2018 /

Published online: 8 October 2018

(C) The Author(s) 2018

\begin{abstract}
In the paper we solve the limit problem for partial maxima of $m$-dependent stationary random fields and we extend the obtained solution to fields satisfying some local mixing conditions. New methods for describing the limitting distribution of maxima are proposed. A notion of a phantom distribution function for a random field is investigated. As an application, several original formulas for calculation of the extremal index are provided. Moving maxima and moving averages as well as Gaussian fields satisfying the Berman condition are considered.
\end{abstract}

Keywords Stationary random fields $\cdot$ Extremes $\cdot m$-dependence $\cdot$ Extremal index · Phantom distribution function · Moving averages · Moving maxima $\cdot$ Berman's condition

AMS 2000 Subject Classifications $60 \mathrm{G} 70 \cdot 60 \mathrm{G} 60 \cdot$ 60F05

\section{Introduction}

Leadbetter and Rootzén (1998) showed that the class of limit distributions for suitably centered and normalized partial maxima of stationary weakly dependent random fields coincides with the corresponding class for i.i.d. sequences. This observation, however, does not mean that also the normalizing and centering constants are the same as in the i.i.d case, for they may be heavily dependent on the local structure of the random field under consideration.

Natalia Soja-Kukieła

natas@mat.umk.pl

Adam Jakubowski

adjakubo@mat.umk.pl

1 Faculty of Mathematics and Computer Science, Nicolaus Copernicus University, ul. Chopina 12/18, 87-100 Toruń, Poland 
For stationary weakly dependent sequences this phenomenon has been well understood for long time and can be easily demonstrated within the simplest non-i.i.d. class of $m$-dependent stationary random variables (Newell 1964). So it is rather surprising that there seems to be no existing limit theory for $m$-dependent stationary random fields. To the authors' knowledge the only attempts in this direction have been made by Turkman (2006) and Ferreira and Pereira (2008), but their results do not yield any concise method of calculating the limits and the extremal index for $m$-dependent random fields. In particular, the formula proposed in the latter paper does not work for the simple 1-dependent random field given in Example 5.5 below.

In Section 2 we study the impact of local dependencies on asymptotics of maxima by means of the Bonferroni-like inequality due to Jakubowski and Rosiński (1999). Theorem 2.1 obtained this way completely explains the $m$-dependent case in a way analogous (but not identical) to Newell's (1964) result for sequences.

The developed machinery proves to be equally effective in a substantially larger class of stationary random fields. In Proposition 2.3 we provide a condition similar to Condition $D^{(m+1)}\left(v_{n}\right)$ of Chernick et al. (1991) guaranteeing that the asymptotics of partial maxima of a random field can be determined on the base of tail properties of joint distribution of a fixed finite dimension (like in the case of $m$-dependent random fields). Section 3.2 serves appropriate non- $m$-dependent examples.

Another form of local dependency is exhibited by moving averages or moving maxima, for which detailed calculations can be found in Basrak and Tafro (2014). The asymptotics of maxima or the extremal index of such random fields involve infinitely many parameters, that cannot, in general, be deduced from any fixed finite dimensional distribution. Nevertheless the maxima of the original random field can be approximated by maxima of suitable $m$-dependent random fields and the limit parameters for the original random field can be obtained as limits of parameters of the approximating sequence.

The above general scheme is very close in spirit to so called $L^{p}$ - $m$-approximability, a notion formally introduced in Hörmann and Kokoszka (2010), but possessing both long history and important applications. Of course, the idea of $L^{p}-m$-approximability, originally created for sums, could not be directly adopted to the needs of the limit theory for maxima of random fields. Therefore we first propose the proper notion of max-m-approximability in Definition 2.4, and then, in Theorem 2.5, we show that our abstract framework works. We also show that both moving averages and moving maxima fit with our formalism (see Section 3.1).

In Section 4 we define and discuss the notion of a phantom distribution function for random fields. Phantom distribution functions for sequences, closely related to extremal indices, were introduced by O'Brien (1987) as follows. For $\left\{X_{n}: n \in \mathbb{Z}\right\}$, a stationary sequence with partial maxima $M_{n}:=\max \left\{X_{k}: 1 \leq k \leq n\right\}$, we say that any distribution function $G$ satisfying

$$
\sup _{x \in \mathbb{R}}\left|P\left(M_{n} \leq x\right)-G(x)^{n}\right| \rightarrow 0, \quad \text { as } \quad n \rightarrow \infty,
$$

is a phantom distribution function. In the special case, when condition (1) holds with $G(x)=P\left(X_{0} \leq x\right)^{\theta}$ for some $\theta \in(0,1]$, we call $\theta$ the extremal index, following Leadbetter (1983). It is worthy to note that sometimes a phantom distribution function 
exists while the extremal index $\theta \in(0,1]$ does not (see, e.g., Doukhan et al. 2015 and references therein). We refer to Doukhan et al. (2015) for the recent results on existence of phantom distribution functions.

Sections 5 and 6 are devoted to extremal indices of random fields. Basing on the results presented in Sections 2 and 4, we provide formulas for the extremal index for some classes of fields. Then we apply these formulas to calculate the extremal index for moving maxima and moving averages. It is also shown that the extremal index of a centered stationary Gaussian field satisfying the Berman condition equals 1.

In the paper, we consider a $d$-dimensional stationary random field $\left\{X_{\mathbf{n}}: \mathbf{n} \in \mathbb{Z}^{d}\right\}$ and its partial maxima of the form

$$
M_{\mathbf{n}}:=\max \left\{X_{\mathbf{k}}: \mathbf{1} \leq \mathbf{k} \leq \mathbf{n} \text { coordinatewise }\right\} .
$$

An element $\mathbf{n} \in \mathbb{Z}^{d}$ is often denoted by $\left(n_{1}, n_{2}, \ldots, n_{d}\right)$; we write $n^{*}:=n_{1} n_{2} \cdots n_{d}$ and $\|\mathbf{n}\|$ is the sup norm; we put $\mathbf{0}:=(0,0, \ldots, 0), \mathbf{1}:=(1,1, \ldots, 1), \infty:=$ $(\infty, \infty, \ldots, \infty)$.

\section{Maxima of random fields satisfying some local conditions}

In the forthcoming section we investigate the asymptotic behaviour of maxima under different local mixing conditions. First we present a limit theorem for $m$-dependent case. Then we extend the obtained result to some other classes of random fields. The notion of max- $m$-approximability is introduced.

\subsection{Asymptotics for maxima of $\boldsymbol{m}$-dependent fields and some generalization}

We recall that $\left\{X_{\mathbf{n}}\right\}$ is $m$-dependent for some $m \in \mathbb{N}$, if families $\left\{X_{\mathbf{n}}: \mathbf{n} \in A\right\}$ and $\left\{X_{\mathbf{k}}: \mathbf{k} \in B\right\}$ are independent for every pair of finite sets $A, B \subset \mathbb{Z}^{d}$ satisfying

$$
\min _{\mathbf{n} \in A, \mathbf{k} \in B}\|\mathbf{n}-\mathbf{k}\|>m,
$$

where $\|\mathbf{n}-\mathbf{k}\|=\max \left\{\left|n_{1}-k_{1}\right|,\left|n_{2}-k_{2}\right|, \ldots,\left|n_{d}-k_{d}\right|\right\}$.

In the following, we will assume that $\left\{X_{\mathbf{n}}\right\}$ is $m$-dependent and the condition

$$
\limsup _{n \rightarrow \infty} n^{d} P\left(X_{\mathbf{0}}>v_{n}\right)<\infty
$$

holds for some sequence $\left\{v_{n}\right\} \subset \mathbb{R}$. It is not difficult to show that then for $\mathbf{N}(n) \rightarrow \infty$ satisfying $N^{*}(n):=N_{1}(n) N_{2}(n) \cdots N_{d}(n)=O\left(n^{d}\right)$ we have

$$
P\left(M_{\mathbf{N}(n)} \leq v_{n}\right)=P\left(M_{\left(\left\lfloor N_{1}(n) / k_{n}\right\rfloor,\left\lfloor N_{2}(n) / k_{n}\right\rfloor, \ldots,\left\lfloor N_{d}(n) / k_{n}\right\rfloor\right)} \leq v_{n}\right)^{k_{n}^{d}}+o(1)
$$

for every sequence $\left\{k_{n}\right\} \subset \mathbb{N}$ tending to infinity such that $k_{n}=o\left(N_{l}(n)\right)$ for each $l$, with $\lfloor\cdot\rfloor$ the floor function. Combining the above equality with the classical fact (see, e.g., O’Brien 1987):

$$
\left(a_{n}\right)^{n}-\exp \left(-n\left(1-a_{n}\right)\right) \rightarrow 0 \quad \text { as } \quad n \rightarrow \infty, \quad \text { for } \quad a_{n} \in[0,1]
$$


we obtain that

$P\left(M_{\mathbf{N}(n)} \leq v_{n}\right)=\exp \left(-k_{n}^{d} P\left(M_{\left(\left\lfloor N_{1}(n) / k_{n}\right\rfloor,\left\lfloor N_{2}(n) / k_{n}\right\rfloor, \ldots,\left\lfloor N_{d}(n) / k_{n}\right\rfloor\right)}>v_{n}\right)\right)+o(1)$.

Theorem 2.1 Assume that the stationary field $\left\{X_{\mathbf{n}}\right\}$ is $m$-dependent and (2) holds with some $\left\{v_{n}\right\} \subset \mathbb{R}$.

(a) If $\mathbf{N}(n) \rightarrow \infty$ is such that $N^{*}(n)=O\left(n^{d}\right)$, then

$$
P\left(M_{\mathbf{N}(n)} \leq v_{n}\right)=\exp \left(-N^{*}(n) \cdot D\left(m, v_{n}\right)\right)+o(1)
$$

with $D(m, v)$ defined as

$$
D(m, v):=\sum_{\boldsymbol{\varepsilon} \in\{0,1\}^{d}}(-1)^{\varepsilon_{1}+\varepsilon_{2}+\ldots+\varepsilon_{d}} P\left(M_{(m+1, m+1, \ldots, m+1)-\varepsilon}>v\right) .
$$

(b) If the condition

$$
\limsup _{n \rightarrow \infty} P\left(M_{(n, n, \ldots, n)} \leq v_{n}\right)<1
$$

is satisfied, then the asymptotic behaviour (4) holds for every $\mathbf{N}(n) \rightarrow \infty$.

Proof To prove (a), let us consider $\mathbf{N}(n) \rightarrow \infty$ and $T>0$ such that $N^{*}(n) \leq T n^{d}$. Then (3) holds, provided that $k_{n} \rightarrow \infty$ slowly. Applying the Bonferroni-type inequality given by Theorem 2.1 of Jakubowski and Rosiński (1999), we can approximate the exponent in (3) as follows

$$
\begin{aligned}
& \left|k_{n}^{d} P\left(M_{\left(\left\lfloor N_{1}(n) / k_{n}\right\rfloor,\left\lfloor N_{2}(n) / k_{n}\right\rfloor, \ldots,\left\lfloor N_{d}(n) / k_{n}\right\rfloor\right)}>v_{n}\right)-N^{*}(n) D\left(m, v_{n}\right)\right| \\
& \leq a(m) \cdot k_{n} \sum_{l=1}^{d} \frac{N^{*}(n)}{N_{l}(n)} P\left(X_{\mathbf{0}}>v_{n}\right) \cdot(1+o(1)) \\
& \quad+b(m) \cdot k_{n}^{d} \sum_{\mathbf{i}, \mathbf{j} \in \prod_{l=1}^{d}\left\{1,2, \ldots,\left\lfloor N_{l}(n) / k_{n}\right\rfloor\right\},}^{\substack{i \mathbf{i}-\mathbf{j} \|>m \\
\text { (7) }}} P\left(X_{\mathbf{i}}>v_{n}, X_{\mathbf{j}}>v_{n}\right)+o(1)=: R(n)
\end{aligned}
$$

with $a(m):=2^{d+1} m\left((m+1)^{d}-1\right)$ and $b(m):=2^{-1}+2^{d-1}(2 m+1)^{d}$. Using $m$ dependence and other assumptions of the theorem, we conclude that the right-hand side denoted by $R(n)$ satisfies

$$
\begin{aligned}
R(n) \leq a(m) & \cdot \operatorname{Tn}^{d} P\left(X_{\mathbf{0}}>v_{n}\right) \sum_{l=1}^{d} \frac{k_{n}}{N_{l}(n)} \cdot(1+o(1)) \\
& +b(m) \cdot k_{n}^{d}\left(\frac{T n^{d}}{k_{n}^{d}}\right)^{2} P\left(X_{\mathbf{0}}>v_{n}\right)^{2}+o(1)=o(1),
\end{aligned}
$$

whenever $k_{n} \rightarrow \infty$ slowly. Combining the above approximation with equation (3), we complete the proof of part (a) of the theorem.

Now, let us assume that (6) holds. Observe that, since (a) is true, it is sufficient to show that (4) is satisfied for every $\mathbf{N}(n) \rightarrow \infty$ such that $N^{*}(n)=T_{n} n^{d}$ with some 
$T_{n} \rightarrow \infty$, to establish part (b) of the theorem. We will prove, using (a), that both the left and the right sides of (4) tend to zero in this case. Indeed, for the right-hand side we have

$$
\begin{aligned}
\exp \left(-N^{*}(n) \cdot D\left(m, v_{n}\right)\right) & =\exp \left(-T_{n} \cdot n^{d} \cdot D\left(m, v_{n}\right)\right) \\
& =\left(P\left(M_{(n, n, \ldots, n)} \leq v_{n}\right)+o(1)\right)^{T_{n}}=o(1) .
\end{aligned}
$$

To show that the left-hand side also tends to zero, let us consider $\psi(n) \rightarrow \infty$ from Lemma 7.1 and note that

$$
\begin{aligned}
\limsup _{n \rightarrow \infty} P\left(M_{\mathbf{N}(n)} \leq v_{n}\right) & \leq \limsup _{n \rightarrow \infty} P\left(M_{\left.\left(\psi_{1}(n), \psi_{2}(n), \ldots, \psi_{d-1}(n),\left\lfloor T \cdot \psi_{d}(n)\right\rfloor\right) \leq v_{n}\right)}\right. \\
& =\limsup _{n \rightarrow \infty} \exp \left(-T \cdot n^{d} \cdot D\left(m, v_{n}\right)\right) \\
& =\limsup _{n \rightarrow \infty} P\left(M_{(n, n, \ldots, n)} \leq v_{n}\right)^{T}
\end{aligned}
$$

for arbitrary $T>0$, where we applied part (a) of the theorem in the last two relations. Hence we get $P\left(M_{\mathbf{N}(n)} \leq v_{n}\right)=o(1)$ and the proof is complete.

Remark 2.2 As an anonymous reviewer noticed, one can find in Theorem 2.1 the shape of standard compound Poisson approximation theorems (see, e.g., Arratia et al. 1989, Barbour and Chryssaphinou 2001). According to this approach, the random variable

$$
\Lambda_{n}:=\sum_{\mathbf{1} \leq \mathbf{k} \leq \mathbf{N}(n)} \sum_{\boldsymbol{\varepsilon} \in\{0,1\}^{d}}(-1)^{\varepsilon_{1}+\varepsilon_{2}+\ldots+\varepsilon_{d}} \mathbb{1}_{\left\{M_{\mathbf{k}, \mathbf{k}+(m, m, \ldots, m)-\varepsilon}>v_{n}\right\}},
$$

with $M_{\mathbf{k}, \mathbf{n}}:=\max \left\{X_{\mathbf{j}}: \mathbf{k} \leq \mathbf{j} \leq \mathbf{n}\right\}$ and $\lambda_{n}:=E \Lambda_{n}=N^{*}(n) \cdot D\left(m, v_{n}\right)$, approximates the number of clusters of exceedances over $v_{n}$ in $\{\mathbf{j}: \mathbf{1} \leq \mathbf{j} \leq \mathbf{N}(n)\}$.

In fact, one can assume some weaker than $m$-dependence conditions on $\left\{X_{\mathbf{n}}\right\}$ and, by fully analogous arguments to those from the proof of Theorem 2.1, calculate limits for maxima by knowledge of $(m+1)^{d}$ terms.

Proposition 2.3 Assume the stationary field $\left\{X_{\mathbf{n}}\right\}$ fulfills (2) with $\left\{v_{n}\right\} \subset \mathbb{R}$. Suppose that

(i) condition (3) and

(ii) the local mixing condition:

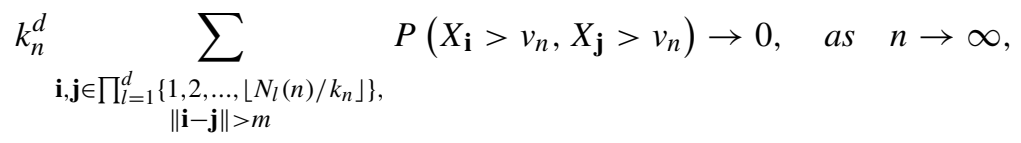

are both satisfied for all $\mathbf{N}(n) \rightarrow \infty$ such that $N^{*}(n)=O\left(n^{d}\right)$, with some $k_{n} \rightarrow \infty$ such that $k_{n}=o\left(N_{l}(n)\right)$ for each $l \in\{1,2, \ldots, d\}$. Then statements $(a)$ and $(b)$ from Theorem 2.1 hold. 
Proof We mime the proof of Theorem 2.1. To approximate the exponent in the righthand side of equality (3) (guaranteed by (i)) we apply Theorem 2.1 of Jakubowski and Rosiński (1999). It is crucial to observe that the right-hand side of (7) tends to zero due to local condition (ii).

Note that assumption (8) is in the same spirit as Condition $D^{(m+1)}\left(v_{n}\right)$ proposed by Chernick et al. (1991). Examples of non-m-dependent fields fulfilling (8) are given in Section 3.2.

\subsection{Asymptotics of maxima of max- $m$-approximable fields}

Definition 2.4 Suppose that the stationary field $\left\{X_{\mathbf{n}}\right\}$ satisfies condition (2) with some $\left\{v_{n}\right\} \subset \mathbb{R}$. We call $\left\{X_{\mathbf{n}}\right\}$ max-m-approximable if it admits the representation

$$
X_{\mathbf{n}}:=f\left(\left\{Z_{\mathbf{n}+\mathbf{j}}: \mathbf{j} \in \mathbb{Z}^{d}\right\}\right), \quad \text { for } \quad \mathbf{n} \in \mathbb{Z}^{d},
$$

with $\left\{Z_{\mathbf{j}}: \mathbf{j} \in \mathbb{Z}^{d}\right\}$ a family of i.i.d. random variables and $f: \mathbb{R}^{\mathbb{Z}^{d}} \rightarrow \mathbb{R}$ a measurable function, and, moreover, for $\delta(m, v)$ defined as

$$
\delta(m, v):=P\left(f\left(\left\{Z_{\mathbf{j}}: \mathbf{j} \in \mathbb{Z}^{d}\right\}\right) \leq v, f\left(\left\{Z_{\mathbf{j}}:\|\mathbf{j}\| \leq m\right\} \cup\left\{Z_{\mathbf{j}}^{\prime}:\|\mathbf{j}\|>m\right\}\right)>v\right),
$$

with $\left\{Z_{\mathbf{j}}^{\prime}\right\}$ an independent copy of $\left\{Z_{\mathbf{j}}\right\}$, the following property

$$
\limsup _{m \rightarrow \infty} \limsup _{n \rightarrow \infty} n^{d} \delta\left(m, v_{n}\right)=0
$$

holds.

Investigating max- $m$-approximable fields, we will often deal with the case when there exists a sequence $r_{n} \rightarrow \infty$ such that $a\left(m_{n}, n\right) \rightarrow 0$, as $n \rightarrow \infty$, holds for every $m_{n} \rightarrow \infty$ satisfying $m_{n} \leq r_{n}$, for some $a(m, n) \in \mathbb{R}$. In this case we will say that $a\left(m_{n}, n\right) \rightarrow 0$ for all $m_{n} \rightarrow \infty$ sufficiently slowly.

From Lemma 7.2 we know that assumption (10) ensures that $n^{d} \delta\left(m_{n}, v_{n}\right) \rightarrow 0$, as $n \rightarrow \infty$, for $m_{n} \rightarrow \infty$ sufficiently slowly.

In fact, partial maxima $M_{\mathbf{n}}$ of max- $m$-approximable $\left\{X_{\mathbf{n}}\right\}$ may be approximated by maxima $M_{\mathbf{n}}^{[m]}$ of the field $\left\{X_{\mathbf{n}}^{[m]}\right\}$ defined for $m \in \mathbb{N}$ as follows

$$
X_{\mathbf{n}}^{[m]}:=f\left(\left\{Z_{\mathbf{n}+\mathbf{j}}:\|\mathbf{j}\| \leq m\right\} \cup\left\{Z_{\mathbf{n}+\mathbf{j}}^{(\mathbf{n})}:\|\mathbf{j}\|>m\right\}\right), \quad \text { for } \quad \mathbf{n} \in \mathbb{Z}^{d},
$$

where $\left\{Z_{\mathbf{j}}^{(\mathbf{n})}: \mathbf{j} \in \mathbb{Z}^{d}\right\}$, for $\mathbf{n} \in \mathbb{Z}^{d}$, are independent, also of $\left\{Z_{\mathbf{j}}\right\}$, copies of $\left\{Z_{\mathbf{j}}\right\}$. The field $\left\{X_{\mathbf{n}}^{[m]}\right\}$ is $(2 m)$-dependent and stationary, moreover, has the same marginal distribution as $\left\{X_{\mathbf{n}}\right\}$. Observe that applying the elementary inequality

$$
\left|P\left(\bigcap_{\mathbf{1} \leq \mathbf{n} \leq \mathbf{N}(n)} A_{\mathbf{n}}\right)-P\left(\bigcap_{\mathbf{1} \leq \mathbf{n} \leq \mathbf{N}(n)} B_{\mathbf{n}}\right)\right| \leq \sum_{\mathbf{1} \leq \mathbf{n} \leq \mathbf{N}(n)} P\left(A_{\mathbf{n}} \triangle B_{\mathbf{n}}\right),
$$


with $\triangle$ the symmetric difference, for $A_{\mathbf{n}}:=\left\{X_{\mathbf{n}} \leq v_{n}\right\}$ and $B_{\mathbf{n}}:=\left\{X_{\mathbf{n}}^{[m]} \leq v_{n}\right\}$, then using stationarity of the fields $\left\{X_{\mathbf{n}}\right\}$ and $\left\{X_{\mathbf{n}}^{[m]}\right\}$ and the fact that random vectors $\left(X_{\mathbf{0}}, X_{\mathbf{0}}^{[m]}\right)$ and $\left(X_{\mathbf{0}}^{[m]}, X_{\mathbf{0}}\right)$ are equal in distribution, we obtain that

$$
\begin{aligned}
& \left|P\left(M_{\mathbf{N}(n)} \leq v_{n}\right)-P\left(M_{\mathbf{N}(n)}^{[m]} \leq v_{n}\right)\right| \\
& \leq N^{*}(n) P\left(X_{\mathbf{0}} \leq v_{n}, X_{\mathbf{0}}^{[m]}>v_{n}\right)+N^{*}(n) P\left(X_{\mathbf{0}}>v_{n}, X_{\mathbf{0}}^{[m]} \leq v_{n}\right) \\
& =2 N^{*}(n) P\left(X_{\mathbf{0}} \leq v_{n}, X_{\mathbf{0}}^{[m]}>v_{n}\right) \\
& =2 N^{*}(n) P\left(f\left(\left\{Z_{\mathbf{j}}: \mathbf{j} \in \mathbb{Z}^{d}\right\}\right) \leq v_{n}, f\left(\left\{Z_{\mathbf{j}}:\|\mathbf{j}\| \leq m\right\} \cup\left\{Z_{\mathbf{j}}^{\prime}:\|\mathbf{j}\|>m\right\}\right)>v_{n}\right) \\
& =2 N^{*}(n) \delta\left(m, v_{n}\right) .
\end{aligned}
$$

Note that for $N^{*}(n)=O\left(n^{d}\right)$ and $m_{n} \rightarrow \infty$ sufficiently slowly, under assumption (10), the right-hand side of (11) tends to zero.

Theorem 2.5 Assume that the stationary field $\left\{X_{\mathbf{n}}\right\}$ fulfills (2) and (10) with some sequence $\left\{v_{n}\right\} \subset \mathbb{R}$.

(a) If $\mathbf{N}(n) \rightarrow \infty$ satisfies $N^{*}(n)=O\left(n^{d}\right)$, then

$$
P\left(M_{\mathbf{N}(n)} \leq v_{n}\right)=\exp \left(-N^{*}(n) \cdot \tilde{D}\left(2 m_{n}, v_{n}\right)\right)+o(1)
$$

for every sequence $\left\{m_{n}\right\} \subset \mathbb{N}$ tending to infinity sufficiently slowly, with $\tilde{D}(2 m, v)$ given by

$$
\tilde{D}(2 m, v):=\sum_{\varepsilon \in\{0,1\}^{d}}(-1)^{\varepsilon_{1}+\varepsilon_{2}+\ldots+\varepsilon_{d}} P\left(M_{(2 m+1,2 m+1, \ldots, 2 m+1)-\varepsilon}^{[m]}>v\right) .
$$

(b) If the condition

$$
\limsup _{n \rightarrow \infty} P\left(M_{(n, n, \ldots, n)} \leq v_{n}\right)<1
$$

holds, then (12) is true for all $\mathbf{N}(n) \rightarrow \infty$.

Proof To prove (a), let us consider $\mathbf{N}(n) \rightarrow \infty$ such that $N^{*}(n) \leq T n^{d}$ for some $T>0$. Then, by (11), we know that

$$
\left|P\left(M_{\mathbf{N}(n)} \leq v_{n}\right)-P\left(M_{\mathbf{N}(n)}^{[m]} \leq v_{n}\right)\right| \leq 2 T n^{d} \delta\left(m, v_{n}\right) .
$$

Since $X_{\mathbf{0}} \stackrel{d}{=} X_{\mathbf{0}}^{[m]}$,

$$
\limsup _{n \rightarrow \infty} n^{d} P\left(X_{\mathbf{0}}^{[m]}>v_{n}\right)=\limsup _{n \rightarrow \infty} n^{d} P\left(X_{\mathbf{0}}>v_{n}\right)<\infty
$$

holds. Applying Theorem 2.1 for the field $\left\{X_{\mathbf{n}}^{[m]}\right\}$, we obtain

$$
P\left(M_{\mathbf{N}(n)}^{[m]} \leq v_{n}\right)-\exp \left(-N^{*}(n) \cdot \tilde{D}\left(2 m, v_{n}\right)\right)=o(1) .
$$


Combining the above results with (10) gives

$$
\begin{aligned}
& \limsup _{m \rightarrow \infty} \limsup _{n \rightarrow \infty}\left|P\left(M_{\mathbf{N}(n)} \leq v_{n}\right)-\exp \left(-N^{*}(n) \cdot \tilde{D}\left(2 m, v_{n}\right)\right)\right| \\
& \leq \limsup _{m \rightarrow \infty} \limsup _{n \rightarrow \infty}\left|P\left(M_{\mathbf{N}(n)} \leq v_{n}\right)-P\left(M_{\mathbf{N}(n)}^{[m]} \leq v_{n}\right)\right| \\
& +\limsup _{m \rightarrow \infty} \limsup _{n \rightarrow \infty}\left|P\left(M_{\mathbf{N}(n)}^{[m]} \leq v_{n}\right)-\exp \left(-N^{*}(n) \cdot \tilde{D}\left(2 m, v_{n}\right)\right)\right| \\
& \leq \limsup \lim \sup 2 T n^{d} \delta\left(m, v_{n}\right)+0 \\
& =0 \text {. }
\end{aligned}
$$

By Lemma 7.2, it follows that

$$
\lim _{n \rightarrow \infty}\left|P\left(M_{\mathbf{N}(n)} \leq v_{n}\right)-\exp \left(-N^{*}(n) \cdot \tilde{D}\left(2 m_{n}, v_{n}\right)\right)\right|=0
$$

for every $m_{n} \rightarrow \infty$ sufficiently slowly. This completes the proof of (a). By analogous arguments to those from the proof of Theorem 2.1(b), one can show that also part (b) holds.

Corollary 2.6 If (2) and (10) are satisfied and, moreover,

$$
\lim _{n \rightarrow \infty} P\left(M_{(n, n, \ldots, n)}^{[m]} \leq v_{n}\right)=\gamma_{m} \quad \text { and } \quad \lim _{m \rightarrow \infty} \gamma_{m}=\gamma
$$

for some $\gamma, \gamma_{1}, \gamma_{2}, \ldots \in(0,1)$, then

$$
\lim _{n \rightarrow \infty} P\left(M_{(n, n, \ldots, n)} \leq v_{n}\right)=\gamma
$$

Corollary 2.7 If (2) and (10) hold and, moreover, D and $\tilde{D}$ given by (5) and (13), respectively, satisfy

$$
\limsup _{m \rightarrow \infty} \limsup _{n \rightarrow \infty} N^{*}(n) \cdot\left|\tilde{D}\left(2 m, v_{n}\right)-D\left(2 m, v_{n}\right)\right|=0
$$

then, for every sequence $\left\{m_{n}\right\} \subset \mathbb{N}$ tending to infinity sufficiently slowly,

$$
P\left(M_{\mathbf{N}(n)} \leq v_{n}\right)=\exp \left(-N^{*}(n) \cdot D\left(2 m_{n}, v_{n}\right)\right)+o(1)
$$

Property (14) is guaranteed by, e.g., the strengthened condition (10):

$$
\limsup _{m \rightarrow \infty} \limsup _{n \rightarrow \infty} m^{d} n^{d} \delta\left(m, v_{n}\right)=0
$$

In Section 3.1 we show that both moving maxima and moving averages belong to the class of max- $m$-approximable fields. 


\section{Examples: random fields fulfilling local conditions}

\subsection{Moving maxima and moving averages as max-m-approximable fields}

We will show that random fields of moving maxima and moving averages, built on fields of i.i.d. regular variables, are max- $m$-approximable.

In the following, $\left\{Z_{\mathbf{n}}\right\}$ is an array of i.i.d. random variables satisfying for some index $\alpha>0$ and slowly varying function $L$

$$
P\left(\left|Z_{\mathbf{0}}\right|>x\right)=x^{-\alpha} L(x)
$$

and the tail balance condition

$$
\frac{P\left(Z_{\mathbf{0}}>x\right)}{P\left(\left|Z_{\mathbf{0}}\right|>x\right)}=p \quad \text { as } \quad x \rightarrow \infty, \quad \text { for some } \quad p \in[0,1],
$$

is assumed. We define $a_{n}:=\inf \left\{y>0: P\left(\left|Z_{\mathbf{0}}\right|>y\right) \leq n^{-d}\right\}$ and $v_{n}:=a_{n} v$ with some fixed $v>0$. Then we have

$$
n^{d} P\left(\left|Z_{\mathbf{0}}\right|>v_{n}\right) \rightarrow v^{-\alpha} \quad \text { as } n \rightarrow \infty
$$

For definitions of moving maxima and moving averages, we will need some weights $c_{\mathbf{j}} \in \mathbb{R}, \mathbf{j} \in \mathbb{Z}^{d}$, satisfying appropriate conditions. The trivial case $c_{\mathbf{j}}=0$ for all $\mathbf{j} \in \mathbb{Z}^{d}$ is excluded.

\subsubsection{Moving maxima}

Let $\left\{X_{\mathbf{n}}\right\}$ be defined by (9) with $f$ given by $f\left(\left\{z_{\mathbf{j}}: \mathbf{j} \in \mathbb{Z}^{d}\right\}\right):=\max _{\mathbf{j} \in \mathbb{Z}^{d}} c_{\mathbf{j}} z_{\mathbf{j}}$, i.e.

$$
X_{\mathbf{n}}=\max _{\mathbf{j} \in \mathbb{Z}^{d}} c_{\mathbf{j}} Z_{\mathbf{n}+\mathbf{j}},
$$

where

$$
\sum_{\mathbf{j} \in \mathbb{Z}^{d}}\left|c_{\mathbf{j}}\right|^{\beta}<\infty \text { for some } 0<\beta<\alpha .
$$

Then $\left\{X_{\mathbf{n}}\right\}$ is well defined and

$$
\lim _{x \rightarrow \infty} \frac{P\left(\max _{\mathbf{j} \in \mathbb{Z}^{d}}\left|c_{\mathbf{j}} Z_{\mathbf{j}}\right|>x\right)}{P\left(\left|Z_{\mathbf{0}}\right|>x\right)}=\lim _{x \rightarrow \infty} \frac{1-\prod_{\mathbf{j} \in \mathbb{Z}^{d}} P\left(\left|c_{\mathbf{j}} Z_{\mathbf{j}}\right| \leq x\right)}{P\left(\left|Z_{\mathbf{0}}\right|>x\right)}=\sum_{\mathbf{j} \in \mathbb{Z}^{d}}\left|c_{\mathbf{j}}\right|^{\alpha}<\infty,
$$

due to Lemma 2.2 from Cline (1983). Moreover, as a corollary, we obtain that the convergence

$$
\lim _{x \rightarrow \infty} \frac{P\left(X_{\mathbf{0}}>x\right)}{P\left(\left|Z_{\mathbf{0}}\right|>x\right)}=\lim _{x \rightarrow \infty} \frac{P\left(\max _{\mathbf{j} \in \mathbb{Z}^{d}} c_{\mathbf{j}} Z_{\mathbf{j}}>x\right)}{P\left(\left|Z_{\mathbf{0}}\right|>x\right)}=p \sum_{c_{\mathbf{j}}>0} c_{\mathbf{j}}^{\alpha}+q \sum_{c_{\mathbf{j}}<0}\left|c_{\mathbf{j}}\right|^{\alpha}
$$

with $q:=1-p$ holds. 
The field $\left\{X_{\mathbf{n}}\right\}$, called the moving maximum field, is max- $m$-approximable. Indeed, applying the above properties, we obtain that

$$
\begin{aligned}
\limsup _{n \rightarrow \infty} n^{d} \delta\left(m, v_{n}\right) & \leq \limsup _{n \rightarrow \infty} n^{d} P\left(\max _{\|\mathbf{j}\|>m} c_{\mathbf{j}} Z_{\mathbf{j}}>v_{n}\right) \\
& \leq \limsup _{n \rightarrow \infty} n^{d} P\left(\max _{\|\mathbf{j}\|>m}\left|c_{\mathbf{j}} Z_{\mathbf{j}}\right|>v_{n}\right) \\
& =\limsup _{n \rightarrow \infty} n^{d} P\left(\left|Z_{\mathbf{0}}\right|>v_{n}\right) \sum_{\|\mathbf{j}\|>m}\left|c_{\mathbf{j}}\right|^{\alpha} \\
& =v^{-\alpha} \sum_{\|\mathbf{j}\|>m}\left|c_{\mathbf{j}}\right|^{\alpha} .
\end{aligned}
$$

Since the right-hand side tends to zero as $m \rightarrow \infty$, condition (10) holds.

\subsubsection{Moving averages}

Consider $\left\{X_{\mathbf{n}}\right\}$ defined by (9) with $f$ given by $f\left(\left\{z_{\mathbf{j}}: \mathbf{j} \in \mathbb{Z}^{d}\right\}\right):=\sum_{\mathbf{j} \in \mathbb{Z}^{d}} c_{\mathbf{j}} z_{\mathbf{j}}$, i.e.

$$
X_{\mathbf{n}}=\sum_{\mathbf{j} \in \mathbb{Z}^{d}} c_{\mathbf{j}} Z_{\mathbf{j}}
$$

where

$$
\sum_{\mathbf{j} \in \mathbb{Z}^{d}}\left|c_{\mathbf{j}}\right|^{\beta}<\infty \text { for some } 0<\beta<\min \{\alpha, 1\} .
$$

Then, due to Theorems 2.1 and 2.3 from Cline (1983), the field $\left\{X_{\mathbf{n}}\right\}$ is well defined and we have

$$
\lim _{x \rightarrow \infty} \frac{P\left(\left|X_{\mathbf{0}}\right|>x\right)}{P\left(\left|Z_{\mathbf{0}}\right|>x\right)}=\lim _{x \rightarrow \infty} \frac{P\left(\left|\sum_{\mathbf{j} \in \mathbb{Z}^{d}} c_{\mathbf{j}} Z_{\mathbf{j}}\right|>x\right)}{P\left(\left|Z_{\mathbf{0}}\right|>x\right)}=\sum_{\mathbf{j} \in \mathbb{Z}^{d}}\left|c_{\mathbf{j}}\right|^{\alpha}<\infty .
$$

Moreover, by Lemma A3.26 from Embrechts et al. (2003),

$$
\lim _{x \rightarrow \infty} \frac{P\left(X_{\mathbf{0}}>x\right)}{P\left(\left|Z_{\mathbf{0}}\right|>x\right)}=\lim _{x \rightarrow \infty} \frac{P\left(\sum_{\mathbf{j} \in \mathbb{Z}^{d}} c_{\mathbf{j}} Z_{\mathbf{j}}>x\right)}{P\left(\left|Z_{\mathbf{0}}\right|>x\right)}=p \sum_{c_{\mathbf{j}}>0} c_{\mathbf{j}}^{\alpha}+q \sum_{c_{\mathbf{j}}<0}\left|c_{\mathbf{j}}\right|^{\alpha} .
$$

It will be shown that $\left\{X_{\mathbf{n}}\right\}$, called the moving average field, is max-m-approximable. First, we note that

$$
\begin{aligned}
n^{d} \delta\left(m, v_{n}\right) \leq n^{d} P\left(\sum_{\|\mathbf{j}\| \leq m} c_{\mathbf{j}} Z_{\mathbf{j}}>v_{n}, \sum_{\mathbf{j} \in \mathbb{Z}^{d}} c_{\mathbf{j}} Z_{\mathbf{j}} \leq v_{n}\right) \\
\quad+n^{d} P\left(\sum_{\|\mathbf{j}\| \leq m} c_{\mathbf{j}} Z_{\mathbf{j}} \leq v_{n}, \sum_{\|\mathbf{j}\|>m} c_{\mathbf{j}} Z_{\mathbf{j}}^{\prime}>v_{n}-\sum_{\|\mathbf{j}\| \leq m} c_{\mathbf{j}} Z_{\mathbf{j}}\right) \\
=: R_{1}\left(m, v_{n}\right)+R_{2}\left(m, v_{n}\right),
\end{aligned}
$$


with $\left\{Z_{\mathbf{n}}^{\prime}\right\}$ an independent copy of $\left\{Z_{\mathbf{n}}\right\}$. For $m \in \mathbb{N}$, let us choose $t_{m}>0$ so that

$$
\left(t_{m}\right)^{2 \alpha}=\sum_{\|\mathbf{j}\|>m}\left|c_{\mathbf{j}}\right|^{\alpha} .
$$

For the first summand in (20), we have

$$
\begin{aligned}
R_{1}\left(m, v_{n}\right)= & n^{d} P\left(\sum_{\|\mathbf{j}\| \leq m} c_{\mathbf{j}} Z_{\mathbf{j}}>v_{n}, \sum_{\|\mathbf{j}\|>m} c_{\mathbf{j}} Z_{\mathbf{j}} \leq v_{n}-\sum_{\|\mathbf{j}\| \leq m} c_{\mathbf{j}} Z_{\mathbf{j}}\right) \\
\leq & n^{d} P\left(\sum_{\|\mathbf{j}\| \leq m} c_{\mathbf{j}} Z_{\mathbf{j}}>\left(1+t_{m}\right) v_{n}, \sum_{\|\mathbf{j}\|>m} c_{\mathbf{j}} Z_{\mathbf{j}} \leq v_{n}-\sum_{\|\mathbf{j}\| \leq m} c_{\mathbf{j}} Z_{\mathbf{j}}\right) \\
& +n^{d} P\left(\left(1+t_{m}\right) v_{n} \geq \sum_{\|\mathbf{j}\| \leq m} c_{\mathbf{j}} Z_{\mathbf{j}}>v_{n}, \sum_{\|\mathbf{j}\|>m} c_{\mathbf{j}} Z_{\mathbf{j}} \leq v_{n}-\sum_{\|\mathbf{j}\| \leq m} c_{\mathbf{j}} Z_{\mathbf{j}}\right) \\
\leq & n^{d} P\left(\sum_{\|\mathbf{j}\|>m} c_{\mathbf{j}} Z_{\mathbf{j}}<-t_{m} v_{n}\right)+n^{d} P\left(\left(1+t_{m}\right) v_{n} \geq \sum_{\|\mathbf{j}\| \leq m} c_{\mathbf{j}} Z_{\mathbf{j}}>v_{n}\right) \\
\leq & n^{d} P\left(\left|\sum_{\|\mathbf{j}\|>m} c_{\mathbf{j}} Z_{\mathbf{j}}\right|>t_{m} v_{n}\right)+n^{d} P\left(\left(1+t_{m}\right) v_{n} \geq \mid \sum_{\|\mathbf{j}\| \leq m} c_{\mathbf{j}} Z_{\mathbf{j}}>v_{n}\right) .
\end{aligned}
$$

Then, by property (18) and the choice of $v_{n}$, we conclude that for all $m \in \mathbb{N}$

$$
\begin{aligned}
R_{1}\left(m, v_{n}\right) \leq & n^{d} P\left(\left|Z_{\mathbf{0}}\right|>t_{m} v_{n}\right) \sum_{\|\mathbf{j}\|>m}\left|c_{\mathbf{j}}\right|^{\alpha} \\
& +n^{d}\left(P\left(\left|Z_{\mathbf{0}}\right|>v_{n}\right)-P\left(\left|Z_{\mathbf{0}}\right|>\left(1+t_{m}\right) v_{n}\right)\right) \sum_{\|\mathbf{j}\| \leq m}\left|c_{\mathbf{j}}\right|^{\alpha}+o(1) \\
= & v^{-\alpha} t_{m}^{-\alpha} \sum_{\|\mathbf{j}\|>m}\left|c_{\mathbf{j}}\right|^{\alpha}+v^{-\alpha}\left(1-\left(1+t_{m}\right)^{-\alpha}\right) \sum_{\|\mathbf{j}\| \leq m}\left|c_{\mathbf{j}}\right|^{\alpha}+o(1),
\end{aligned}
$$

as $n \rightarrow \infty$. Using similar arguments for the second summand in (20), we get

$$
\begin{aligned}
R_{2}\left(m, v_{n}\right)= & n^{d} P\left(\sum_{\|\mathbf{j}\| \leq m} c_{\mathbf{j}} Z_{\mathbf{j}} \leq\left(1-t_{m}\right) v_{n}, \sum_{\|\mathbf{j}\|>m} c_{\mathbf{j}} Z_{\mathbf{j}}^{\prime}>v_{n}-\sum_{\|\mathbf{j}\| \leq m} c_{\mathbf{j}} Z_{\mathbf{j}}\right) \\
& +n^{d} P\left(\left(1-t_{m}\right) v_{n}<\sum_{\|\mathbf{j}\| \leq m} c_{\mathbf{j}} Z_{\mathbf{j}} \leq v_{n}, \sum_{\|\mathbf{j}\|>m} c_{\mathbf{j}} Z_{\mathbf{j}}^{\prime}>v_{n}-\sum_{\|\mathbf{j}\| \leq m} c_{\mathbf{j}} Z_{\mathbf{j}}\right) \\
\leq & n^{d} P\left(\sum_{\|\mathbf{j}\|>m} c_{\mathbf{j}} Z_{\mathbf{j}}^{\prime}>t_{m} v_{n}\right)+n^{d} P\left(\left(1-t_{m}\right) v_{n}<\sum_{\|\mathbf{j}\| \leq m} c_{\mathbf{j}} Z_{\mathbf{j}} \leq v_{n}\right) \\
\leq & n^{d} P\left(\left|\sum_{\|\mathbf{j}\|>m} c_{\mathbf{j}} Z_{\mathbf{j}}^{\prime}\right|>t_{m} v_{n}\right)+n^{d} P\left(\left(1-t_{m}\right) v_{n}<\left|\sum_{\|\mathbf{j}\| \leq m} c_{\mathbf{j}} Z_{\mathbf{j}}\right| \leq v_{n}\right) .
\end{aligned}
$$


Applying (18) and the definition of $v_{n}$ again, we obtain that

$$
\begin{aligned}
R_{2}\left(m, v_{n}\right) \leq & n^{d} P\left(\left|Z_{\mathbf{0}}\right|>t_{m} v_{n}\right) \sum_{\|\mathbf{j}\|>m}\left|c_{\mathbf{j}}\right|^{\alpha} \\
& +n^{d}\left(P\left(\left|Z_{\mathbf{0}}\right|>\left(1-t_{m}\right) v_{n}\right)-P\left(\left|Z_{\mathbf{0}}\right|>v_{n}\right)\right) \sum_{\|\mathbf{j}\| \leq m}\left|c_{\mathbf{j}}\right|^{\alpha}+o(1) \\
= & v^{-\alpha} t_{m}^{-\alpha} \sum_{\|\mathbf{j}\|>m}\left|c_{\mathbf{j}}\right|^{\alpha}+v^{-\alpha}\left(\left(1-t_{m}\right)^{-\alpha}-1\right) \sum_{\|\mathbf{j}\| \leq m}\left|c_{\mathbf{j}}\right|^{\alpha}+o(1)
\end{aligned}
$$

as $n \rightarrow \infty$. Summarizing,

$$
\begin{aligned}
\limsup _{n \rightarrow \infty} n^{d} \delta\left(m, v_{n}\right) \leq & \limsup _{n \rightarrow \infty}\left(R_{1}\left(m, v_{n}\right)+R_{2}\left(m, v_{n}\right)\right) \\
\leq & 2 v^{-\alpha} t_{m}^{-\alpha} \sum_{\|\mathbf{j}\|>m}\left|c_{\mathbf{j}}\right|^{\alpha} \\
& +v^{-\alpha}\left(\left(1-t_{m}\right)^{-\alpha}-\left(1+t_{m}\right)^{-\alpha}\right) \sum_{\mathbf{j} \in \mathbb{Z}^{d}}\left|c_{\mathbf{j}}\right|^{\alpha} .
\end{aligned}
$$

To complete the proof, let us observe that the choice of $t_{m}$ entails that the right-hand side of the above inequality tends to zero, as $m \rightarrow \infty$, which implies (10).

\subsection{Weakly dependent Gaussian field and moving maxima built on it}

In this section, we give three examples of random fields which are not $m$-dependent for any $m \in \mathbb{N}$, but satisfy the local condition (8) with some $m \in \mathbb{N}$.

\subsubsection{Weakly dependent Gaussian field}

Let $\left\{W_{\mathbf{n}}: \mathbf{n} \in \mathbb{Z}^{d}\right\}$ be a centered stationary Gaussian random field with correlation $r(\mathbf{n}):=\operatorname{Cov}\left(W_{\mathbf{0}}, W_{\mathbf{n}}\right)$ satisfying the multidimensional Berman condition

$$
r(\mathbf{n}) \cdot \log \|\mathbf{n}\| \rightarrow 0 \quad \text { as } \quad\|\mathbf{n}\| \rightarrow \infty
$$

We denote $\Phi(x):=P\left(W_{0} \leq x\right)$ and $\bar{\Phi}(x):=1-\Phi(x)$. The following adaptation of Lemma 4.3.2 in Leadbetter et al. (1983) will play an important role.

Lemma 3.1 Let $r(\mathbf{n})$ defined above satisfy (21) and let a sequence $\left\{u_{n}: n \in \mathbb{N}\right\} \subset \mathbb{R}$ be such that $\left\{n^{d} \bar{\Phi}\left(u_{n}\right): n \in \mathbb{N}\right\}$ is bounded. Then

$$
n^{d} \sum_{\mathbf{j} \in \prod_{l=1}^{d}\left\{-N_{l}(n), \ldots, N_{l}(n)\right\} \backslash\{\mathbf{0}\}}\left|r_{\max }(\mathbf{j})\right| \exp \left(-\frac{u_{n}^{2}}{1+\left|r_{\max }(\mathbf{j})\right|}\right) \rightarrow 0, \quad \text { as } \quad n \rightarrow \infty,
$$

for every $\mathbf{N}(n)=O\left(n^{d}\right)$, with $r_{\max }(\mathbf{j}):=\max \{r(\mathbf{n}):\|\mathbf{n}\| \geq\|\mathbf{j}\|\}$. 
Let $\left\{v_{n}\right\}$ be chosen so that $\limsup _{n \rightarrow \infty} n^{d} P\left(W_{\mathbf{0}}>v_{n}\right)<\infty$. Put $X_{\mathbf{n}}:=W_{\mathbf{n}}$. Then (3) holds for every $\mathbf{N}(n) \rightarrow \infty$ with some $k_{n} \rightarrow \infty$. Moreover, condition (8) is satisfied with $m=0$. We omit the proof of these facts since they can be easily deduced from considerations (involving Lemma 3.1) presented in Section 3.2.3.

It follows that the extremal index of the weakly dependent Gaussian field $\left\{W_{\mathbf{n}}\right\}$ exists and equals 1; for details see Section 6.3.

\subsubsection{Moving maxima built on a weakly dependent Gaussian field}

Consider $\left\{W_{\mathbf{n}}\right\}$ the Gaussian field from Section 3.2.1. We define $\left\{X_{\mathbf{n}}: \mathbf{n} \in \mathbb{Z}^{d}\right\}$ as

$$
X_{\mathbf{n}}:=\max \left\{c_{\mathbf{j}} W_{\mathbf{n}+\mathbf{j}}: \mathbf{0} \leq \mathbf{j} \leq(m, m, \ldots, m)\right\},
$$

for some $c_{\mathbf{j}} \in\{-1,0,1\}$ and $m \in \mathbb{N}$, and assume that $c_{\max }:=\max \left\{\left|c_{\mathbf{j}}\right|\right\}=1$.

Let $\left\{v_{n}\right\}$ satisfy $\limsup _{n \rightarrow \infty} n^{d} P\left(W_{\mathbf{0}}>v_{n}\right)<\infty$. Then (3) holds for each $\mathbf{N}(n) \rightarrow \infty$ with some $k_{n} \rightarrow \infty$ and (8) is true with $m$. The proof of this fact follows from the results of Section 3.2.3.

\subsubsection{Moving maxima built on a transformed weakly dependent Gaussian field}

Let $\left\{W_{\mathbf{n}}\right\}$ be the Gaussian field from Section 3.2.1. For fixed $\alpha>0$ and for $h: \mathbb{R} \rightarrow$ $\mathbb{R}$ an increasing, odd, bijective function given by

$$
h(x):= \begin{cases}(2 \bar{\Phi}(x))^{-1 / \alpha}-1, & \text { if } x \geq 0 \\ 1-(2 \Phi(x))^{-1 / \alpha} & , \text { if } x<0\end{cases}
$$

we define $\left\{Z_{\mathbf{n}}: \mathbf{n} \in \mathbb{Z}^{d}\right\}$ by $Z_{\mathbf{n}}:=h\left(W_{\mathbf{n}}\right)$. Observe that then we have

$$
P\left(Z_{0}>x\right)=P\left(Z_{0}<-x\right)=\frac{(1+x)^{-\alpha}}{2}, \text { for any } x>0 \text {, }
$$

and hence conditions (15) and (16) are fulfilled.

We define $\left\{X_{\mathbf{n}}: \mathbf{n} \in \mathbb{Z}^{d}\right\}$ as $X_{\mathbf{n}}:=\max \left\{c_{\mathbf{j}} Z_{\mathbf{n}+\mathbf{j}}: \mathbf{0} \leq \mathbf{j} \leq(m, m, \ldots, m)\right\}$, for some $c_{\mathbf{j}} \in \mathbb{R}$, and assume that $c_{\max }:=\max \left\{\left|c_{\mathbf{j}}\right|\right\}>0$.

Let a sequence $\left\{v_{n}\right\} \subset \mathbb{R}$ be chosen so that $\lim _{n \rightarrow \infty} n^{d} P\left(Z_{\mathbf{0}}>v_{n}\right)<\infty$ holds. Then the condition $\limsup _{n \rightarrow \infty} n^{d} \bar{\Phi}\left(u_{n}^{(c)}\right)<\infty$ is also satisfied for $u_{n}^{(c)}:=$ $h^{-1}\left(v_{n} / c\right)$, for every $c>0$.

From the results for moving maxima recalled in Section 3.1.1 we know that if $\left\{Z_{\mathbf{n}}\right\}$ were independent then (2) would be true. To prove property (2) in the considered weakly dependent Gaussian setting, one can apply The Normal Comparison Lemma (see, e.g., Theorem 4.2.1 and its Corollary 4.2.4 in Leadbetter et al. 1983).

The field $\left\{X_{\mathbf{n}}\right\}$ defined above fulfills conditions (3) and (8) with $m$, for each $\mathbf{N}(n) \rightarrow \infty$ with some $k_{n} \rightarrow \infty$. Both of them may be successfully verified by use of Lemma 3.1 (compare with the proof of Lemma 4.4.1 in Leadbetter et al. 1983). 
Here we shall show (8). In order to do this, let us consider arbitrary $\mathbf{N}(n) \rightarrow \infty$ such that $N^{*}(n)=O\left(n^{d}\right)$ and $k_{n} \rightarrow \infty$. Observe that for large $n$ we have

$$
\begin{aligned}
& k_{n}^{d} \quad \sum \quad P\left(X_{\mathbf{i}}>v_{n}, X_{\mathbf{j}}>v_{n}\right) \\
& \mathbf{i}, \mathbf{j} \in \prod_{l=1}^{d}\left\{1,2, \ldots,\left\lfloor N_{l}(n) / k_{n}\right\rfloor\right\}, \\
& \leq C_{1} n^{d} \quad \sum \quad P\left(X_{\mathbf{j}}>v_{n}, X_{\mathbf{0}}>v_{n}\right) \\
& \mathbf{j} \in \prod_{l=1}^{d}\left\{-\left\lfloor N_{l}(n) / k_{n}\right\rfloor, \ldots,\left\lfloor N_{l}(n) / k_{n}\right\rfloor\right\}, \\
& \|\mathbf{j}\|>m
\end{aligned}
$$

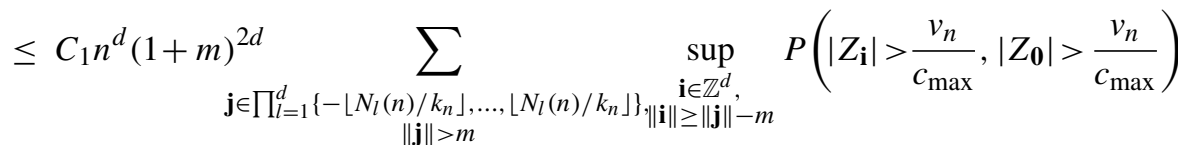

$$
\begin{aligned}
& \leq C_{2} n^{d} \sum_{\mathbf{j} \in \prod_{l=1}^{d}\left\{-\left\lfloor N_{l}(n) / k_{n}\right\rfloor, \ldots,\left\lfloor N_{l}(n) / k_{n}\right\rfloor\right\},} \sup _{\substack{\mathbf{i} \in \mathbf{Z}\left\|\mathbb{Z}^{d},\\
\right\| \mathbf{i} \| \geq m}} P\left(\left|W_{\mathbf{i} \|-m}\right|>u_{n}^{\left(c_{\max }\right)},\left|W_{\mathbf{0}}\right|>u_{n}^{\left(c_{\max }\right)}\right) \\
& \leq C_{3} n^{d} \frac{n^{d}}{k_{n}^{d}} \bar{\Phi}\left(u_{n}^{\left(c_{\max }\right)}\right)^{2} \\
& +C_{4} n^{d} \sum_{\mathbf{j} \in \prod_{l=1}^{d}\left\{-\left\lfloor N_{l}(n) / k_{n}\right\rfloor, \ldots,\left\lfloor N_{l}(n) / k_{n}\right\rfloor\right\},} \sup _{\substack{\mathbf{i} \in \mathbb{Z}^{d},\|\mathbf{j}\|>m}}|r(\mathbf{i})| \exp \left(-\frac{\left(u_{n}^{\left(c_{\max }\right)}\right)^{2}}{1+|r(\mathbf{i})|}\right) \\
& \leq \frac{C_{5}}{k_{n}^{d}}+C_{6} n^{d} \sum_{\mathbf{j} \in \prod_{l=1}^{d}\left\{-N_{l}(n), \ldots, N_{l}(n)\right\} \backslash\{\mathbf{0}\}}\left|r_{\max }(\mathbf{j})\right| \exp \left(-\frac{\left(u_{n}^{\left(c_{\max }\right)}\right)^{2}}{1+\left|r_{\max }(\mathbf{j})\right|}\right),
\end{aligned}
$$

for some constants $C_{1}, C_{2}, C_{3}, C_{4}, C_{5}, C_{6}>0$, where the first three relations follow from the definition of $\left\{X_{\mathbf{n}}\right\}$, the fourth relation is a consequence of Corollary 4.2.4 in Leadbetter et al. (1983) and in the last one we use the fact that $n^{d} \bar{\Phi}\left(u_{n}^{\left(c_{\max }\right)}\right)$ is bounded. Finally, since Lemma 3.1 implies that the right-hand side tends to zero as $n \rightarrow \infty$, condition (8) holds.

\section{Phantom distribution function}

In this section a notion of a phantom distribution function is introduced and some consequences of the results from Section 2 are concluded.

Definition 4.1 We call any distribution function $G$ a phantom distribution function for $\left\{X_{\mathbf{n}}\right\}$, whenever

$$
\sup _{x \in \mathbb{R}}\left|P\left(M_{\mathbf{n}} \leq x\right)-G(x)^{n_{1} n_{2} \cdots n_{d}}\right| \rightarrow 0 \quad \text { as } \quad \mathbf{n} \rightarrow \infty \quad \text { coordinatewise. }
$$


We will assume that the field $\left\{X_{\mathbf{n}}\right\}$ satisfies the following condition

$$
P\left(M_{\psi(n)} \leq v_{n}\right) \rightarrow \gamma \quad \text { as } n \rightarrow \infty, \text { for some } \gamma \in(0,1),
$$

with $\left\{v_{n}\right\} \subset \mathbb{R}$ a nondecreasing sequence, where

$$
\boldsymbol{\psi}=\{\boldsymbol{\psi}(n)\}=\left\{\left(\psi_{1}(n), \psi_{2}(n), \ldots, \psi_{d}(n)\right)\right\} \subset \mathbb{N}^{d}
$$

is a fixed sequence such that

$$
\psi(n) \rightarrow \infty \text { and } \psi^{*}(n):=\psi_{1}(n) \psi_{2}(n) \cdots \psi_{d}(n) \sim n^{d} .
$$

Remark 4.2 If $\left\{X_{\mathbf{n}}\right\}$ is $m$-dependent or max-m-approximable and satisfies (23), then condition (2) holds.

Proof Observe that for $m$-dependent $\left\{X_{\mathbf{n}}\right\}$ we have

$$
P\left(M_{\psi(n)} \leq v_{n}\right) \leq P\left(X_{\mathbf{0}} \leq v_{n}\right)^{\prod_{i=1}^{d}\left\lfloor\psi_{i}(n) /(m+1)\right\rfloor}=\exp \left(-n^{d} P\left(X_{\mathbf{0}}>v_{n}\right) \frac{1+o(1)}{(m+1)^{d}}\right)
$$

and the remark easily follows. If $\left\{X_{\mathbf{n}}\right\}$ is max- $m$-approximable, then (23) combined with (11) entails that maxima of the $(2 m)$-dependent field $\left\{X_{\mathbf{n}}^{[m]}\right\}$ satisfy

$$
0<\liminf _{n \rightarrow \infty} P\left(M_{\psi(n)}^{[m]} \leq v_{n}\right) \leq \limsup _{n \rightarrow \infty} P\left(M_{\psi(n)}^{[m]} \leq v_{n}\right)<1,
$$

for every large $m$. By already used arguments, keeping in mind that $X_{\mathbf{0}}$ and $X_{\mathbf{0}}^{[m]}$ are equal in distribution, we obtain

$$
\limsup _{n \rightarrow \infty} n^{d} P\left(X_{\mathbf{0}}>v_{n}\right)=\limsup _{n \rightarrow \infty} n^{d} P\left(X_{\mathbf{0}}^{[m]}>v_{n}\right)<\infty
$$

and thus (2) is satisfied.

Both (23) and (24) provide the following construction of a candidate for a phantom distribution function for $\left\{X_{\mathbf{n}}\right\}$ :

$$
G(x):= \begin{cases}0 & \text { for } \quad x<v_{1} \\ \gamma^{1 / n^{d}} & \text { for } \quad x \in\left[v_{n}, v_{n+1}\right) \\ 1 & \text { for } \quad x \geq v_{\infty}:=\sup \left\{v_{n}: n \in \mathbb{N}\right\} .\end{cases}
$$

If $d=1$, the above formula reduces to the recipe for the phantom distribution function given in Theorem 1.3 of Jakubowski (1991) (see also O'Brien 1987 and Jakubowski 1993). Observe that $G$ defined by (25) is regular in the sense of O'Brien (1974), i.e.

$$
G\left(G_{*}-\right)=1 \quad \text { and } \quad \lim _{x \rightarrow G_{*}-} \frac{1-G(x-)}{1-G(x)}=1,
$$

with $G_{*}$ the right end of $G$. One should also notice that $G$ is strictly tail-equivalent to its continuous modification $\tilde{G}$ that can be defined similarly as in Theorem 2 by Doukhan et al. (2015).

Using the results from Section 2, we establish the following theorem on phantom distribution functions. 
Theorem 4.3 If $\left\{X_{\mathbf{n}}\right\}$ satisfies (23) and

(i) fulfills the assumptions of Proposition 2.3, in particular if it is m-dependent, or

(ii) is max-m-approximable,

then $G$ given by formula (25) is a phantom distribution function for $\left\{X_{\mathbf{n}}\right\}$.

Proof Let us assume (23) and (i). Observe that it is sufficient to show that

$$
P\left(M_{\mathbf{N}(n)} \leq x_{n}\right)-G\left(x_{n}\right)^{N^{*}(n)} \rightarrow 0, \quad \text { as } \quad n \rightarrow \infty,
$$

holds, whenever $\mathbf{N}(n) \rightarrow \infty$ and $\left\{x_{n}\right\} \subset \mathbb{R}$ is an arbitrary nondecreasing sequence. Note that both in the case $\lim _{n \rightarrow \infty} x_{n}<v_{\infty}$ and when $\lim _{n \rightarrow \infty} x_{n}>v_{\infty}$ convergence (26) is obvious.

Let us consider $\mathbf{N}(n) \rightarrow \infty$ and nondecreasing $x_{n} \rightarrow v_{\infty}$. Let $k\left(x_{n}\right) \in \mathbb{N}$ be chosen so that $v_{k\left(x_{n}\right)} \leq x_{n}<v_{k\left(x_{n}\right)+1}$. Then $k\left(x_{n}\right) \rightarrow \infty$ and we have

$$
P\left(M_{\mathbf{N}(n)} \leq v_{k\left(x_{n}\right)}\right) \leq P\left(M_{\mathbf{N}(n)} \leq x_{n}\right) \leq P\left(M_{\mathbf{N}(n)} \leq v_{k\left(x_{n}\right)+1}\right) .
$$

By Proposition 2.3 we obtain

$$
\begin{aligned}
P\left(M_{\mathbf{N}(n)} \leq v_{k\left(x_{n}\right)}\right) & =\exp \left(-N^{*}(n) \cdot D\left(m, v_{k\left(x_{n}\right)}\right)\right)+o(1) \\
& =\left(\exp \left(-k\left(x_{n}\right)^{d} \cdot D\left(m, v_{k\left(x_{n}\right)}\right)\right)\right)^{N^{*}(n) / k\left(x_{n}\right)^{d}}+o(1) \\
& =\gamma^{N^{*}(n) / k\left(x_{n}\right)^{d}}+o(1) .
\end{aligned}
$$

Similarly, one can show that

$$
P\left(M_{\mathbf{N}(n)} \leq v_{k\left(x_{n}\right)+1}\right)=\gamma^{N^{*}(n) /\left(k\left(x_{n}\right)+1\right)^{d}}+o(1) .
$$

Since $\gamma^{N^{*}(n) /\left(k\left(x_{n}\right)+1\right)^{d}}-\gamma^{N^{*}(n) / k\left(x_{n}\right)^{d}}=o(1)$, we conclude that

$$
P\left(M_{\mathbf{N}(n)} \leq x_{n}\right)=\gamma^{N^{*}(n) / k\left(x_{n}\right)^{d}}+o(1)=G\left(x_{n}\right)^{N^{*}(n)}+o(1)
$$

and thus (26) holds.

By similar arguments to those above, applying Theorem 2.5, one can prove the theorem under assumption (ii).

Remark 4.4 One could also investigate, instead of (22), the convergence of maxima along a fixed sequence $\mathbf{N}(n) \rightarrow \infty$ (compare with Theorem 4.1 in Leadbetter and Rootzén 1998) and consider directional phantom distribution functions. We do not know if there exists a stationary field with a directional phantom distribution function which is not its phantom distribution function in the sense of Definition 4.1.

\section{Extremal index}

In this section we combine results obtained in Sections 2 and 4 to establish new formulas for calculation of the extremal index for random fields. 
Definition 5.1 We say that $\theta \in(0,1]$ is the extremal index for $\left\{X_{\mathbf{n}}\right\}$, if the function $G$ given by $G(x):=P\left(X_{\mathbf{0}} \leq x\right)^{\theta}, x \in \mathbb{R}$, is a phantom distribution function for $\left\{X_{\mathbf{n}}\right\}$.

We note here that some definition of the extremal index for random fields was also proposed by Choi (2002).

Applying some results established in previous sections, we immediately obtain the following theorem for a class including $m$-dependent fields.

Theorem 5.2 Suppose that the stationary field $\left\{X_{\mathbf{n}}\right\}$ satisfies the assumptions of Proposition 2.3 and $n^{d} P\left(X_{\mathbf{0}}>v_{n}\right) \rightarrow \tau$ holds with some $\tau>0$. Let $\theta \in(0,1]$. Then the following three conditions are equivalent.

(a) The number $\theta$ is the extremal index for $\left\{X_{\mathbf{n}}\right\}$.

(b) Relation (23) holds with $\gamma:=\exp (-\theta \tau)$ and some $\psi$ satisfying (24).

(c) The statement

$$
\frac{\sum_{\boldsymbol{\varepsilon} \in\{0,1\}^{d}}(-1)^{\varepsilon_{1}+\varepsilon_{2}+\ldots+\varepsilon_{d}} P\left(M_{(m+1, m+1, \ldots, m+1)-\varepsilon}>v_{n}\right)}{P\left(X_{\mathbf{1}}>v_{n}\right)} \rightarrow \theta, \quad \text { as } n \rightarrow \infty,
$$

is true.

Proof Let $\left\{X_{\mathbf{n}}\right\}$ satisfy the assumptions of the theorem. Observe that condition (a) implies

$$
\begin{aligned}
P\left(M_{\psi(n)} \leq v_{n}\right) & =P\left(X_{\mathbf{0}} \leq v_{n}\right)^{\theta \psi^{*}(n)}+o(1) \\
& =\exp \left(-\theta n^{d} P\left(X_{\mathbf{0}}>v_{n}\right)\right)+o(1)=\exp (-\theta \tau)+o(1)
\end{aligned}
$$

for every $\psi$ satisfying (24) and thus (b) follows. If (b) holds, then from Theorem 4.3 we get that $G$ given by (25) is a phantom distribution function for $\left\{X_{\mathbf{n}}\right\}$. Applying the theorem again we obtain that $\hat{G}(x):=G(x)^{1 / \theta}$ is a phantom distribution function for the field $\left\{\hat{X}_{\mathbf{n}}\right\}$ of i.i.d. random variables with $\hat{X}_{\mathbf{0}} \stackrel{d}{=} X_{\mathbf{0}}$. Then we have

$$
\begin{aligned}
& \sup _{x \in \mathbb{R}}\left|P\left(M_{\mathbf{n}} \leq x\right)-P\left(X_{\mathbf{0}} \leq x\right)^{\theta n_{1} n_{2} \cdots n_{d}}\right| \\
& \leq \sup _{x \in \mathbb{R}}\left|P\left(M_{\mathbf{n}} \leq x\right)-G(x)^{n_{1} n_{2} \cdots n_{d}}\right|+\sup _{x \in \mathbb{R}}\left|\left(\hat{G}(x)^{n_{1} n_{2} \cdots n_{d}}\right)^{\theta}-\left(P\left(X_{\mathbf{0}} \leq x\right)^{n_{1} n_{2} \cdots n_{d}}\right)^{\theta}\right| \\
& =o(1) .
\end{aligned}
$$

Hence $P\left(X_{\mathbf{0}} \leq x\right)^{\theta}, x \in \mathbb{R}$, is a phantom distribution for $\left\{X_{\mathbf{n}}\right\}$ and condition (a) holds. The equivalence of (b) and (c) is due to Proposition 2.3.

Similarly, we can prove the following theorem for max- $m$-approximable fields.

Theorem 5.3 Let the stationary field $\left\{X_{\mathbf{n}}\right\}$ satisfy $n^{d} P\left(X_{\mathbf{0}}>v_{n}\right) \rightarrow \tau$ with some $\tau>0$ and be max-m-approximable. Then conditions $(a),(b)$ from Theorem 5.2 and (c') given below are equivalent. 
(c') We have, as $n \rightarrow \infty$,

$$
\frac{\sum_{\boldsymbol{\varepsilon} \in\{0,1\}^{d}}(-1)^{\varepsilon_{1}+\varepsilon_{2}+\ldots+\varepsilon_{d}} P\left(M_{\left(2 m_{n}+1,2 m_{n}+1, \ldots, 2 m_{n}+1\right)-\varepsilon}^{\left[m_{n}\right]}>v_{n}\right)}{P\left(X_{1}>v_{n}\right)} \rightarrow \theta,
$$

for every $\left\{m_{n}\right\} \subset \mathbb{N}$ tending to infinity sufficiently slowly.

Proof The proof is fully analogous to the argumentation from Theorem 5.2. Here, one shall use Theorem 2.5 instead of Proposition 2.3.

Remark 5.4 For $d=1$ Theorem 5.2 gives the formula:

$$
\theta=\lim _{n \rightarrow \infty} \frac{P\left(M_{m+1}>v_{n}\right)-P\left(M_{m}>v_{n}\right)}{P\left(X_{1}>v_{n}\right)},
$$

after simple transformations

$\theta=\lim _{n \rightarrow \infty} \frac{P\left(M_{m+1}>v_{n}\right)-P\left(M_{2, m+1}>v_{n}\right)}{P\left(X_{1}>v_{n}\right)}=\lim _{n \rightarrow \infty} P\left(M_{2, m+1} \leq v_{n} \mid X_{1}>v_{n}\right)$,

with $M_{2, m+1}:=\max \left\{X_{k}: 2 \leq k \leq m+1\right\}$. This is the well known method of calculating the extremal index $\theta$ for sequences satisfiying some local mixing conditions, including $m$-dependent sequences, with the knowledge of the joint distribution of $(m+1)$ consecutive terms (see, e.g., Chernick et al. 1991).

Theorems 5.2 and 5.3 provide formulas for calculation of the extremal index. We present a simple motivating example below. Further examples with the new tools succesfully applied can be found in Section 6.

Example 5.5 Let $\left\{X_{\mathbf{n}}: \mathbf{n} \in \mathbb{Z}^{2}\right\}$ be given as $X_{\mathbf{n}}:=\max \left\{Z_{\left(n_{1}+1, n_{2}\right)}, Z_{\left(n_{1}, n_{2}+1\right)}\right\}$, where $\left\{Z_{\mathbf{n}}: \mathbf{n} \in \mathbb{Z}^{2}\right\}$ is a field of i.i.d. random variables and $n^{2} P\left(Z_{\mathbf{0}}>v_{n}\right) \rightarrow \tau / 2$, as $n \rightarrow \infty$, holds with some $\left\{v_{n}\right\} \subset \mathbb{R}$ and $\tau>0$. Then $\left\{X_{\mathbf{n}}\right\}$ is 1 -dependent and

$$
\begin{gathered}
n^{2} P\left(X_{\mathbf{0}}>v_{n}\right) \rightarrow \tau \\
P\left(M_{(n, n)} \leq v_{n}\right)=P\left(Z_{\mathbf{0}} \leq v_{n}\right)^{n^{2}}+o(1)=e^{-\tau / 2}+o(1) .
\end{gathered}
$$

From the equivalence of (a) and (b) in Theorem 5.2 we obtain that $\theta=1 / 2$ is the extremal index for $\left\{X_{\mathbf{n}}\right\}$. Moreover, the calculation of $\theta$ based on the formula from part (c) of the theorem looks as follows:

$$
\theta=\lim _{n \rightarrow \infty} \frac{7 P\left(Z_{\mathbf{0}}>v_{n}\right)-4 P\left(Z_{\mathbf{0}}>v_{n}\right)-4 P\left(Z_{\mathbf{0}}>v_{n}\right)+2 P\left(Z_{\mathbf{0}}>v_{n}\right)}{2 P\left(Z_{\mathbf{0}}>v_{n}\right)}=\frac{1}{2} .
$$

On the contrary, the method proposed by Ferreira and Pereira (2008) gives $\theta=1$. 


\section{Examples: calculation of extremal indices}

\subsection{Extremal index for moving maxima}

Let $\left\{X_{\mathbf{n}}: \mathbf{n} \in \mathbb{Z}^{2}\right\}$ be the moving maximum field from Section 3.1.1. Let $\left\{v_{n}\right\} \subset \mathbb{R}$ be the sequence defined therein for some $v>0$. Then, as we already know, $\left\{X_{\mathbf{n}}\right\}$ is max- $m$-approximable and

$$
n^{2} P\left(X_{\mathbf{0}}>v_{n}\right) \rightarrow \tau, \quad \text { as } \quad n \rightarrow \infty,
$$

for

$$
\tau:=\left(p \sum_{\mathbf{j} \in \mathbb{Z}^{d}, c_{\mathbf{j}}>0} c_{\mathbf{j}}^{\alpha}+q \sum_{\mathbf{j} \in \mathbb{Z}^{d}, c_{\mathbf{j}}<0}\left|c_{\mathbf{j}}\right|^{\alpha}\right) v^{-\alpha} .
$$

Our goal is to apply Theorem 5.3 to calculate the extremal index for $\left\{X_{\mathbf{n}}\right\}$.

Let $m \in \mathbb{N}$ be arbitrary. Then maxima of the field $\left\{X_{\mathbf{n}}^{[m]}\right\}$, associated with $\left\{X_{\mathbf{n}}\right\}$, satisfy for every $\boldsymbol{\varepsilon}=\left(\varepsilon_{1}, \varepsilon_{2}\right) \in\{\mathbf{0},(0,1),(1,0), \mathbf{1}\}$ the following condition

$$
\begin{aligned}
& P\left(M_{(2 m+1,2 m+1)-\varepsilon}^{[m]}>v_{n}\right) \\
\sim & \sum_{I \in \mathcal{I}_{\varepsilon}} P\left(\max _{\mathbf{i} \in I}\left(c_{\mathbf{i}} Z_{\mathbf{0}}\right)>v_{n}\right)+\left(2 m+1-\varepsilon_{1}\right)\left(2 m+1-\varepsilon_{2}\right) P\left(\max _{\|\mathbf{i}\|>m}\left(c_{\mathbf{i}} Z_{\mathbf{i}}\right)>v_{n}\right),
\end{aligned}
$$

where $\mathcal{I}_{\varepsilon}:=\left\{\left(\mathbf{j}+I_{\varepsilon}\right) \cap I_{\mathbf{0}}: \mathbf{j} \in \mathbb{Z}^{2}\right\}, I_{\boldsymbol{\varepsilon}}:=\left\{\mathbf{j} \in \mathbb{Z}^{2}:(-m,-m) \leq \mathbf{j} \leq(m, m)-\boldsymbol{\varepsilon}\right\}$. Since we have

$$
\left(\mathcal{I}_{\mathbf{0}} \backslash \mathcal{I}_{(0,1)}\right) \backslash\left(\mathcal{I}_{(1,0)} \backslash \mathcal{I}_{1}\right)=\left\{I_{0}\right\}
$$

and, moreover,

$$
\sum_{\boldsymbol{\varepsilon} \in\{0,1\}^{2}}(-1)^{\varepsilon_{1}+\varepsilon_{2}}\left(2 m+1-\varepsilon_{1}\right)\left(2 m+1-\varepsilon_{2}\right)=1
$$

holds, we conclude that

$$
\begin{aligned}
& \frac{\sum_{\varepsilon \in\{0,1\}^{2}}(-1)^{\varepsilon_{1}+\varepsilon_{2}} P\left(M_{(2 m+1,2 m+1)-\varepsilon}^{[m]}>v_{n}\right)}{P\left(X_{\mathbf{1}}>v_{n}\right)} \\
& =\frac{P\left(\max _{\mathbf{i} \in I_{\mathbf{0}}}\left(c_{\mathbf{i}} Z_{\mathbf{0}}\right)>v_{n}\right)+P\left(\max _{\|\mathbf{i}\|>m} c_{\mathbf{i}} Z_{\mathbf{i}}>v_{n}\right)}{P\left(X_{\mathbf{1}}>v_{n}\right)}+o(1) .
\end{aligned}
$$

Applying the results recalled in Section 3.1.1, we obtain

$$
\begin{aligned}
\frac{P\left(\max _{\mathbf{i} \in I_{\mathbf{0}}}\left(c_{\mathbf{i}} Z_{\mathbf{0}}\right)>v_{n}\right)}{P\left(X_{\mathbf{1}}>v_{n}\right)} & =\frac{P\left(\left(\max _{\mathbf{i} \in I_{\mathbf{0}}} c_{\mathbf{i}}^{+}\right) Z_{\mathbf{0}}>v_{n}\right)+P\left(\left(\max _{\mathbf{i} \in I_{\mathbf{0}}} c_{\mathbf{i}}^{-}\right) Z_{\mathbf{0}}<-v_{n}\right)}{P\left(X_{\mathbf{1}}>v_{n}\right)} \\
& =\frac{p \cdot\left(\max _{\|\mathbf{i}\| \leq m} c_{\mathbf{i}}^{+}\right)^{\alpha}+q \cdot\left(\max _{\|\mathbf{i}\| \leq m} c_{\mathbf{i}}^{-}\right)^{\alpha}}{p \cdot \sum_{\mathbf{j} \in \mathbb{Z}^{d}, c_{\mathbf{j}}>0} c_{\mathbf{j}}^{\alpha}+q \cdot \sum_{\mathbf{j} \in \mathbb{Z}^{d}, c_{\mathbf{j}}<0}\left|c_{\mathbf{j}}\right|^{\alpha}}+o(1),
\end{aligned}
$$


with $c_{\mathbf{i}}^{+}:=\max \left\{c_{\mathbf{i}}, 0\right\}$ and $c_{\mathbf{i}}^{-}:=\max \left\{-c_{\mathbf{i}}, 0\right\}$, and

$$
\begin{aligned}
\frac{P\left(\max _{\|\mathbf{i}\|>m} c_{\mathbf{i}} Z_{\mathbf{i}}>v_{n}\right)}{P\left(X_{\mathbf{1}}>v_{n}\right)} & \leq \frac{P\left(\max _{\|\mathbf{i}\|>m}\left|c_{\mathbf{i}} Z_{\mathbf{i}}\right|>v_{n}\right)}{P\left(X_{\mathbf{1}}>v_{n}\right)} \\
& =\frac{\sum_{\|\mathbf{i}\|>m}\left|c_{\mathbf{i}}\right|^{\alpha}}{p \cdot \sum_{\mathbf{j} \in \mathbb{Z}^{d}, c_{\mathbf{j}}>0} c_{\mathbf{j}}^{\alpha}+q \cdot \sum_{\mathbf{j} \in \mathbb{Z}^{d}, c_{\mathbf{j}}<0}\left|c_{\mathbf{j}}\right|^{\alpha}}+o(1) .
\end{aligned}
$$

All the above approximations are for an arbitrary $m \in \mathbb{N}$ and for $n \rightarrow \infty$.

Observe that if $m \rightarrow \infty$, then we get

$$
\lim _{m \rightarrow \infty} \lim _{n \rightarrow \infty} \frac{\sum_{\varepsilon \in\{0,1\}^{2}}(-1)^{\varepsilon_{1}+\varepsilon_{2}} P\left(M_{(2 m+1,2 m+1)-\varepsilon}^{[m]}>v_{n}\right)}{P\left(X_{1}^{[m]}>v_{n}\right)}=\theta
$$

with

$$
\theta:=\frac{p\left(c^{+}\right)^{\alpha}+q\left(c^{-}\right)^{\alpha}}{p \cdot \sum_{\mathbf{j} \in \mathbb{Z}^{d}, c_{\mathbf{j}} \geq 0} c_{\mathbf{j}}^{\alpha}+q \cdot \sum_{\mathbf{j} \in \mathbb{Z}^{d}, c_{\mathbf{j}}<0}\left|c_{\mathbf{j}}\right|^{\alpha}} \in(0,1],
$$

where $c^{+}:=\max _{\mathbf{i} \in \mathbb{Z}^{2}} c_{\mathbf{i}}^{+}$and $c^{-}:=\max _{\mathbf{i} \in \mathbb{Z}^{2}} c_{\mathbf{i}}^{-}$. By Lemma 7.2, for every sequence $\left\{m_{n}\right\} \subset \mathbb{N}$ tending to infinity sufficiently slowly the convergence from Theorem 5.3 (c') holds. We conclude that the number $\theta$ is the extremal index for $\left\{X_{\mathbf{n}}\right\}$.

\subsection{Extremal index for moving averages}

Let $\left\{X_{\mathbf{n}}: \mathbf{n} \in \mathbb{Z}^{2}\right\}$ be the moving average field from Section 3.1.2, built on $\left\{Z_{\mathbf{n}}\right\}$, with partial maxima denoted by $M(X)_{\mathbf{n}}$. Let $\left\{v_{n}\right\} \subset \mathbb{R}$ be the sequence defined therein for some $v>0$. Then $\left\{X_{\mathbf{n}}\right\}$ is max- $m$-approximable and

$$
n^{2} P\left(X_{\mathbf{0}}>v_{n}\right) \rightarrow \tau, \quad \text { as } n \rightarrow \infty
$$

with $\tau$ given by (27). Below we combine Theorem 5.3 and the result from Section 6.1 to calculate the extremal index for $\left\{X_{\mathbf{n}}\right\}$. In the following, $\left\{Y_{\mathbf{n}}\right\}$ denotes the moving maximum field built on $\left\{Z_{\mathbf{n}}\right\}$ and $M(Y)_{\mathbf{n}}$ are its partial maxima.

For $n \in \mathbb{N}$, we define $A_{n}:=\left\{X_{\mathbf{0}}>v_{n}\right\}=\left\{\sum_{\mathbf{j} \in \mathbb{Z}^{2}} c_{\mathbf{j}} Z_{\mathbf{j}}>v_{n}\right\}$ and $B_{n}:=$ $\left\{Y_{\mathbf{0}}>v_{n}\right\}=\left\{\max _{\mathbf{j} \in \mathbb{Z}^{2}} c_{\mathbf{j}} Z_{\mathbf{j}}>v_{n}\right\}$. Then $n^{2} P\left(A_{n}\right)=n^{2} P\left(B_{n}\right)+o(1)$, as $\rightarrow \infty$, by properties (17) and (19) and the definition of $\left\{v_{n}\right\}$. We will show that also

$$
n^{2} P\left(A_{n} \triangle B_{n}\right) \rightarrow 0, \quad \text { as } n \rightarrow \infty,
$$

is true. To do this, for arbitrary $\varepsilon>0$ we choose $t>0$ so that $P\left(\sum_{\mathbf{i} \in \mathbb{Z}^{2}}\left|c_{\mathbf{i}} Z_{\mathbf{i}}\right|>t\right)<$ $\varepsilon$ and put $B_{n, t}:=\left\{\max _{\mathbf{j} \in \mathbb{Z}^{2}} c_{\mathbf{j}} Z_{\mathbf{j}}>v_{n}+t\right\}$. Then, by the long-tail property guaranteed by regularity (see, e.g., Embrechts et al. 2003, p. 50), $n^{2} P\left(B_{n}\right)=n^{2} P\left(B_{n, t}\right)+o(1)$ holds as $n \rightarrow \infty$. Since $B_{n, t} \subset B_{n}$, it follows that $n^{2} P\left(B_{n} \triangle B_{n, t}\right) \rightarrow 0$. Furthermore,

$$
\begin{aligned}
& n^{2} P\left(B_{n, t} \backslash A_{n}\right) \leq n^{2} \sum_{\mathbf{j} \in \mathbb{Z}^{2}} P\left(c_{\mathbf{j}} Z_{\mathbf{j}}>v_{n}+t, \sum_{\mathbf{i} \in \mathbb{Z}^{2}, \mathbf{i} \neq \mathbf{j}} c_{\mathbf{i}} Z_{\mathbf{i}}<-t\right) \\
& \leq n^{2} \sum_{\mathbf{j} \in \mathbb{Z}^{2}} P\left(c_{\mathbf{j}} Z_{\mathbf{j}}>v_{n}+t\right) \cdot P\left(\sum_{\mathbf{i} \in \mathbb{Z}^{2}}\left|c_{\mathbf{i}} Z_{\mathbf{i}}\right|>t\right) \leq \sum_{\mathbf{j} \in \mathbb{Z}^{2}}\left|c_{\mathbf{j}}\right|^{\alpha} \cdot v^{-\alpha} \cdot(1+o(1)) \cdot \varepsilon .
\end{aligned}
$$


Since $\varepsilon$ is arbitrary and $n^{2} P\left(A_{n}\right)=n^{2} P\left(B_{n, t}\right)+o(1)$, we get $n^{2} P\left(A_{n} \triangle B_{n, t}\right) \rightarrow 0$. We conclude that (29) is satisfied.

Now, observe that condition (29) implies that for every $m \in \mathbb{N}$

$$
\lim _{n \rightarrow \infty} n^{2} P\left(\left\{X_{\mathbf{k}}^{[m]}>v_{n}\right\} \triangle\left\{Y_{\mathbf{k}}^{[m]}>v_{n}\right\}\right)=0,
$$

provided that $X_{\mathbf{k}}^{[m]}$ and $Y_{\mathbf{k}}^{[m]}$ are constructed with the same $\left\{Z_{\mathbf{n}}^{(\mathbf{k})}\right\}$. Moreover, for every $\varepsilon \in\{\mathbf{0},(0,1),(1,0), \mathbf{1}\}$ we have

$$
\begin{aligned}
\limsup _{n \rightarrow \infty} n^{2} P( & \left.\left\{M(X)_{(2 m+1,2 m+1)-\varepsilon}^{[m]}>v_{n}\right\} \triangle\left\{M(Y)_{(2 m+1,2 m+1)-\varepsilon}^{[m]}>v_{n}\right\}\right) \\
\leq & \limsup _{n \rightarrow \infty} n^{2}(2 m+1)^{2} P\left(\left\{X_{\mathbf{0}}^{[m]}>v_{n}\right\} \triangle\left\{Y_{\mathbf{0}}^{[m]}>v_{n}\right\}\right)=0,
\end{aligned}
$$

in particular, as $n \rightarrow \infty$,

$$
n^{2} P\left(M(X)_{(2 m+1,2 m+1)-\varepsilon}^{[m]}>v_{n}\right)=n^{2} P\left(M(Y)_{(2 m+1,2 m+1)-\varepsilon}^{[m]}>v_{n}\right)+o(1) .
$$

Applying the above fact and the result for moving maxima from Section 6.1, we get

$$
\begin{aligned}
& \lim _{m \rightarrow \infty} \lim _{n \rightarrow \infty} \frac{\sum_{\varepsilon \in\{0,1\}^{2}}(-1)^{\varepsilon_{1}+\varepsilon_{2}} P\left(M(X)_{(2 m+1,2 m+1)-\varepsilon}^{[m]}>v_{n}\right)}{P\left(X_{1}>v_{n}\right)} \\
& =\lim _{m \rightarrow \infty} \lim _{n \rightarrow \infty} \frac{\sum_{\varepsilon \in\{0,1\}^{2}}(-1)^{\varepsilon_{1}+\varepsilon_{2}} P\left(M(Y)_{(2 m+1,2 m+1)-\varepsilon}^{[m]}>v_{n}\right)}{P\left(X_{1}>v_{n}\right)}=\theta
\end{aligned}
$$

with $\theta$ given by (28). Since for all sequences $\left\{m_{n}\right\} \subset \mathbb{N}$ tending to infinity sufficiently slowly the convergence from Theorem 5.3(c') holds (see Lemma 7.2), the number $\theta$ is the extremal index for $\left\{X_{\mathbf{n}}\right\}$.

\subsection{Extremal index for a Gaussian field satisfying the Berman condition}

Let $\left\{X_{\mathbf{n}}: \mathbf{n} \in \mathbb{Z}^{d}\right\}$ be a centered stationary Gaussian random field with correlation $r(\mathbf{n})$ satisfying the Berman condition (21). Then the extremal index of $\left\{X_{\mathbf{n}}\right\}$ equals $\theta=1$.

To show it, let us consider $\left\{v_{n}\right\}$ satisfying $\lim \sup _{n \rightarrow \infty} n^{d} P\left(X_{\mathbf{0}}>v_{n}\right) \rightarrow \tau$ with some $\tau>0$. From Section 3.2.1 we know that assumptions (3) and (8) with $m=0$ are fulfilled. Applying Theorem 5.2(c) we get that $\theta=1$ is the extremal index for $\left\{X_{\mathbf{n}}\right\}$.

Acknowledgements The authors thank the reviewers and the associate editor for their suggestions and comments which helped to improve the manuscript.

Open Access This article is distributed under the terms of the Creative Commons Attribution 4.0 International License (http://creativecommons.org/licenses/by/4.0/), which permits unrestricted use, distribution, and reproduction in any medium, provided you give appropriate credit to the original author(s) and the source, provide a link to the Creative Commons license, and indicate if changes were made. 


\section{Appendix}

Two self-serving lemmas applied in the proof of Theorem 2.1 and in Section 2.2 are given below.

Lemma 7.1 Let $\mathbf{N}(n) \rightarrow \infty$ be such that $N^{*}(n) / n^{d} \rightarrow \infty$. Then there exists a sequence $\{\boldsymbol{\psi}(n)\} \subset \mathbb{N}^{d}$ satisfying $\boldsymbol{\psi}(n) \rightarrow \infty, \psi_{1}(n) \psi_{2}(n) \cdots \psi_{d}(n) \sim n^{d}, \psi_{i}(n) \leq$ $N_{i}(n)$ for $i \in\{1,2, \ldots, d-1\}$ and $\psi_{d}(n) / N_{d}(n) \rightarrow 0$.

Proof Let the sequences $\left\{t_{i}(n)\right\} \subset \mathbb{R}$, for $i \in\{1,2, \ldots, d-1\}$, be chosen so that

$$
t_{1}(n) \rightarrow \infty, \quad t_{1}(n)=o\left(n^{d}\right), \quad t_{1}(n)=o\left(N_{2}(n) N_{3}(n) \cdots N_{d}(n)\right),
$$

and

$$
t_{i}(n) \rightarrow \infty, \quad t_{i}(n)=o\left(t_{i-1}(n)\right), \quad t_{i}(n)=o\left(N_{i+1}(n) N_{i+2}(n) \cdots N_{d}(n)\right)
$$

for $2 \leq i \leq d-1$. Let us consider $a_{i}(n) \in \mathbb{R}$ defined as follows

$$
a_{i}(n):= \begin{cases}\min \left\{N_{1}(n), \frac{n^{d}}{t_{1}(n)}\right\} & \text { if } i=1 ; \\ \min \left\{N_{i}(n), \frac{n^{d}}{a_{1}(n) a_{2}(n) \cdots a_{i-1}(n) t_{i}(n)}\right\} & \text { if } i \in\{2,3, \ldots, d-1\} ; \\ \frac{n^{d}}{a_{1}(n) a_{2}(n) \cdots a_{d-1}(n)} & \text { if } i=d .\end{cases}
$$

Then, we easily get that $a_{i}(n) \rightarrow \infty$ and $a_{i}(n) \leq N_{i}(n)$ for $i \in\{1,2, \ldots, d-1\}$. We will show that also $a_{d}(n) \rightarrow \infty$ and $a_{d}(n) / N_{d}(n) \rightarrow 0$. Indeed, by the definition of $a_{d-1}(n), a_{d-2}(n), \ldots$, we have

$$
\begin{aligned}
a_{d}(n) & =\frac{n^{d}}{a_{1}(n) \cdots a_{d-1}(n)} \\
& =\max \left\{\frac{n^{d}}{a_{1}(n) \cdots a_{d-2}(n) N_{d-1}(n)}, t_{d-1}(n)\right\} \\
& =\max \left\{\frac{n^{d}}{a_{1}(n) \cdots a_{d-3}(n) N_{d-2}(n) N_{d-1}(n)}, \frac{t_{d-2}(n)}{N_{d-1}(n)}, t_{d-1}(n)\right\} \\
& =\ldots=\max \left\{\frac{n^{d}}{N_{1}(n) \cdots N_{d-1}(n)}, \max _{2 \leq i \leq d-1} \frac{t_{i-1}(n)}{N_{i}(n) \cdots N_{d-1}(n)}, t_{d-1}(n)\right\} .
\end{aligned}
$$

Since $t_{d-1}(n) \rightarrow \infty$ and $a_{d}(n) \geq t_{d-1}(n)$, the condition $a_{d}(n) \rightarrow \infty$ holds. Moreover, applying $n^{d}=o\left(N^{*}(n)\right)$ and $t_{i-1}(n)=o\left(N_{i}(n) N_{i+1}(n) \cdots N_{d}(n)\right)$, we conclude that $a_{d}(n) / N_{d}(n) \rightarrow 0$. To complete, we shall define $\psi_{i}(n):=\left\lfloor a_{i}(n)\right\rfloor \in \mathbb{N}$.

Lemma 7.2 For $a(m, n) \geq 0, b(m, n) \in \mathbb{R}\left(m \in \mathbb{N}_{+}, n \in \mathbb{N}\right), b \in \mathbb{R}$,

(i) if $\limsup _{m \rightarrow \infty} \limsup _{n \rightarrow \infty} a(m, n)=0$ holds, then $\lim _{n \rightarrow \infty} a\left(m_{n}, n\right)=0$;

(ii) if $\lim _{m \rightarrow \infty} \lim _{n \rightarrow \infty} b(m, n)=b$ holds, then $\lim _{n \rightarrow \infty} b\left(m_{n}, n\right)=b$,

for some sequence $r_{n} \rightarrow \infty$ and all $m_{n} \rightarrow \infty$ satisfying $m_{n} \leq r_{n}$.

Proof To prove (i), observe that for every $m$ and $a(m):=\limsup _{n \rightarrow \infty} a(m, n)$, there exists $N_{m} \in \mathbb{N}$ such that $a(m, n)-a(m) \leqslant 1 / m$ for all $n \geq N_{m}$. Let us choose $N_{m}$ for 
each $m$ so that the sequence $\left\{N_{m}\right\}$ is increasing. Define $r_{n}:=\sum_{k=1}^{\infty} k \cdot \mathbb{1}_{\left[N_{k}, N_{k+1}\right)}(n)$. Then $r_{n} \rightarrow \infty$ and for every $m_{n} \rightarrow \infty$ satisfying $m_{n} \leq r_{n}$ we obtain

$$
a\left(m_{n}, n\right)-a\left(m_{n}\right) \leq \frac{1}{m_{n}},
$$

since $n \geq N_{r_{n}} \geq N_{m_{n}}$ holds, and consequently

$$
0 \leq a\left(m_{n}, n\right)=\left(a\left(m_{n}, n\right)-a\left(m_{n}\right)\right)+a\left(m_{n}\right) \leq \frac{1}{m_{n}}+o(1)=o(1) .
$$

The proof of part (ii) is fully analogous.

\section{References}

Arratia, R., Goldstein, L., Gordon, L.: Two moments suffice for Poisson approximations: the Chen-Stein method. Ann. Probab. 17, 9-25 (1989)

Barbour, A.D., Chryssaphinou, O.: Compound Poisson approximation: a user's guide. Ann. Appl. Probab. 11, 964-1002 (2001)

Basrak, B., Tafro, A.: Extremes of moving averages and moving maxima on a regular lattice. Probab. Math. Statist. 34, 61-79 (2014)

Chernick, M.R., Hsing, T., McCormick, W.P.: Calculating the extremal index for a class of stationary sequences. Adv. Appl. Probab. 23, 835-850 (1991)

Choi, H.: Central Limit Theory and Extremes of Random Fields. PhD dissertation (2002)

Cline, D.: Infinite series of random variables with regularly varying tails. Technical Report 83-24, Institute of Applied Mathematics and Statistics, Univ. British Columbia. Available at www.stat.tamu.edu/ $\sim$ dcline/Papers/infiniteseries.pdf (1983)

Doukhan, P., Jakubowski, A., Lang, G.: Phantom distribution functions for some stationary sequences. Extremes 18, 697-725 (2015)

Embrechts, P., Klüppelberg, C., Mikosch, T.: Modelling Extremal Events for Insurance and Finance. Springer, Berlin (2003)

Ferreira, H., Pereira, L.: How to compute the extremal index of stationary random fields. Statist. Probab. Lett. 78, 1301-1304 (2008)

Hörmann, S., Kokoszka, P.: Weakly dependent functional data. Ann. Statist. 38, 1845-1884 (2010)

Jakubowski, A.: Relative extremal index of two stationary processes. Stochastic Process. Appl. 37, 281297 (1991)

Jakubowski, A.: An asymptotic independent representation in limit theorems for maxima of nonstationary random sequences. Ann. Probab. 21, 819-830 (1993)

Jakubowski, A., Rosiński, J.: Local dependencies in random fields via a Bonferroni type inequality. Contemp. Math. 234, 85-95 (1999). Available also at arXiv:1709.01165

Leadbetter, M.R.: Extremes and local dependence in stationary sequences. Z. Wahrscheinlichkeitstheor. verw. Geb. 65, 291-306 (1983)

Leadbetter, M.R., Lindgren, G., Rootzén, H.: Extremes and Related Properties of Random Sequences and Processes. Springer, New York (1983)

Leadbetter, M.R., Rootzén, H.: On Extreme Values in Stationary Random Fields. In: Karatzas, I., Rajput, B.S., Taqqu, M. (eds.) Stochastic Processes and Related Topics. In Memory of Stamatis Cambanis 1943-1995, pp. 275-285. Birkäuser, Boston (1998)

Newell, G.F.: Asymptotic extremes for $m$-dependent random variables. Ann. Math. Statist. 35, 1322-1325 (1964)

O'Brien, G.: Limit theorems for the maximum term of a stationary process. Ann. Probab. 2, 540-545 (1974)

O’Brien, G.: Extreme values for stationary and Markov sequences. Ann. Probab. 15, 281-291 (1987)

Turkman, K.F.: A note on the extremal index for space-time processes. J. Appl. Prob. 43, 114-126 (2006) 